In Crescendo, 2020; 11(2): 207-224

Fecha de recepción: 13/01/2020

Fecha de aceptación: 23/01/2020

\title{
IMPLEMENTACIÓN DE SISTEMAS INTEGRADOS DE GESTIÓN EN EMPRESAS AREQUIPEÑAS COMO HERRAMIENTAS DE GESTIÓN
}

\author{
IMPLEMENTATION OF INTEGRATED MANAGEMENT SYSTEMS IN \\ AREQUIPA COMPANIES AS MANAGEMENT TOOLS
}

\author{
Walter F. Deza-Loyaga', Jorge A. Aparicio-Ballena², \\ Jenry A. Hidalgo-Lama ${ }^{3}$
}

\section{RESUMEN}

Este artículo muestra resultados de implementación de Sistemas Integrados de Gestión: ISO 9001, ISO 14001, OSHAS 18001; en empresas del Parque Industrial de Arequipa. El objetivo de este estudio, que utiliza un enfoque empírico, es analizar la implementación del sistema integrado de gestión a la totalidad de empresas de este gremio, en la investigación se escogió a ocho empresas a fin de evaluar si tienen una de las tres certificaciones internacionales vigentes, además de realizar un análisis específico. El estudio se realizó entre los meses de junio y noviembre del 2019 a los responsables del Sistema Integrado de Gestión, se aplicó una encuesta y una entrevista. Se concluye que solo 19 empresas situadas en el parque industrial de Arequipa han implementado alguna de las tres certificaciones internacionales. Se confirma que las empresas con alguna certificación tienen mejores condiciones y mejores procesos de gestión que las empresas no certificadas.

PALABRAS ClAVE: Calidad, Sistemas de Gestión, ISO 9001, ISO 14001, OSHAS 18001.

1 Ingeniero Industrial, Docente de la Universidad Católica de Santa María y SENATI. wdezaloy@ucsm.edu.pe, https://orcid.org/0000-0002-4005-0200

2 Licenciado en Administración, Docente de la Universidad César Vallejo y Universidad Tecnológica del Perú.C18730@utp.edu.pe,https://orcid.org/0000-0001-5901-3752

3 Licenciado en Economía, Docente de la Universidad Privada Antenor Orrego. jhidalgol@upao.edu.pe,https://orcid.org/0000-0001-5901-752 


\begin{abstract}
This article shows results of implementation of Integrated Management Systems: ISO 9001, ISO 14001, OSHAS 18001; in companies of the Arequipa Industrial Park. The objective of this study, which uses an empirical approach, is to analyze the implementation of the integrated management system to all the companies of this union, in the research eight companies were chosen in order to evaluate if they have one of the three international certifications current, in addition to carrying out a specific analysis. The study was carried out between the months of June and November 2019 to those responsible for the Integrated Management System, a survey and an interview were applied. It is concluded that only 19 companies located in the Arequipa industrial park have implemented any of the three international certifications. It is confirmed that companies with some certification have better conditions and better management processes than non-certified companies.
\end{abstract}

KEY WORDS: Quality, Management Systems, ISO 9001, ISO 14001, OSHAS 18001.

\title{
INTRODUCCIÓN
}

El documento "Perú: Estructura Empresarial, 2018" del Instituto Nacional de Estadística e Informática (INEI), señala que existen 2393033 empresas en el Perú. Del total de las unidades empresariales en el año 2018, 2 millones 270 mil 423 fueron clasificadas como microempresas (94,9\%), seguido de 100 mil 443 pequeñas empresas (4,2\%), 14 mil 281 grandes y medianas empresas $(0,6 \%)$ y 7 mil 886 que representaron a la administración pública (0,3\%). En Arequipa existen 133 mil 846 empresas. (Instituto Nacional de Estadística e Informática INEI, 2019).

El total de las empresas activas que figuran en la página Web de la Asociación de Empresas del Parque Industrial de Arequipa es de 40, distribuidas en los siguientes rubros: Agroindustrias (01), Automotriz (02), Consumo (03), Educación (03), Financieras (01), Industrial (19), Minería (05), Servicio Social (02), Textil (03), Transporte (01). (ADEPIA, 2019)

Para comprender los conceptos que integran esta investigación, es importante conceptualizar que es un sistema, una gestión, y un sistema integrado de gestión; una vez que tengamos claro estos conceptos es más acertada la definición ideal de sistema integrado de gestión.

- Sistema: Según la Norma ISO 9001 se expresa que un sistema "es un conjunto de elementos mutuamente relacionados o que interactúan". (Calidad ISO 9001, 2019). Y una definición muy general de sistema es 
entendido como "Un objeto complejo cuyos componentes se relacionan con al menos algún otro componente e interactúan entre si con un objetivo, un sistema puede ser material o conceptual". (Jiménez, D., 2019).

- Gestión: "Acciones o diligencias que permiten la realización de una operación organizacional o de anhelo cualquiera. En el mundo empresarial, el término gestión es asociado con la administración de un negocio". (Jiménez, D., 2019).

- Sistemas integrados de gestión: "ayudan a las organizaciones a mejorar permanente la calidad de los productos y servicios, a establecer y evaluar programas, política, control y objetivos. La creación de un sistema de gestión integrado simplifica el desarrollo, mantenimiento y utilidad de varios sistemas de gestión". (ISO TOOLS, 2019).

De acuerdo con los últimos datos recopilados por la Organización Internacional de Normalización (ISO), En la ISO Survey 2018 sacamos que la ISO 9001 de 2015 es la norma con mayor número de certificados expedidos en el mundo, con un total de 878 664. En segundo lugar, con menos de la mitad de los certificados que la ISO 9001, está la ISO 14001:2018 con 307 059. Para el caso de Perú se reporta (en una aproximación oficial) un total de 1350 certificados (válidos) de ISO 9001 y de 465 certificados (válidos) de ISO 14001 en el 2018. —(ISO Survey, 2019).

Geromin, A. y Galleguillo, D. (2015) en la ASSE Professional Development Conference and Exposition, mencionan lo siguiente: "está comprobado que los sistemas de gestión (OSHAS 18001, ISO 9001 y 14001) y su integración en cualquier organización reducen los costos directos e indirectos; Además, estandarizan las operaciones e implementan la estructura".

El estándar ISO 9001 no solo puede considerarse como un paso inicial para la simple conformación a los requisitos de calidad, sino también como un marco para la mejora de la efectividad del sistema de gestión de calidad (SGC) (McGuire y Dilts 2008). El estándar define "Eficacia del SGC" como la medida en que se alcanzan los objetivos previstos (ISO 9001 2008). Van Der Spiegel et al. (2007) y Trigueros, Pina y Sansalvador Selles (2008) también sugieren que el ISO 9001 (QMS) es más efectivo y debería probarse por el grado de evaluación en que se cumplen los estándares de los objetivos preestablecidos. (Evangelos y Jiju, 2014)

La evidencia empírica sobre las consecuencias de desempeño derivadas de la adopción de estos estándares es extensa (Abad, Lafuente y Vilajosana, 2013), y la 
mayoría de los artículos se centran en una u otra categoría de estándares. Por ejemplo, De Vries, Bayramoglu y Van der Wiele (2012) revisaron la literatura existente sobre el impacto de ISO 14001 y demostró que, en general, se documenta una influencia positiva en el desempeño de las empresas. Mientras Gavronski, Ferrer y Paiva (2008) identifican cuatro fuentes de motivación para la adopción de las normas ambientales ISO 14001, Heras-Saizarbitoria, Molina-Azorín y Dick (2011) utilizan un análisis longitudinal de cinco años para comparar el desempeño de los resultados financieros de las empresas en España antes y después de la certificación, y descubren que no evidencian mejoras en el desempeño seguido a la certificación. (Albulescu et al., 2016)

En el marco empresarial actual existe una tendencia creciente a la integración de varios sistemas de gestión, entre los que se destacan la calidad, el medio ambiente, la salud y seguridad en el trabajo, y recientemente el sistema de gestión energética. De esta forma, las organizaciones gestionan sus procesos y brindan productos y servicios con mayor calidad, garantizando la preservación del medio ambiente, la salud y seguridad de sus trabajadores y mayor exigencia e integralidad en el control y sostenibilidad de sus recursos energéticos. (Antúnez Saiz, 2016).

La flexibilidad del sistema integrado de gestión, a partir de los diferentes fundamentos del estudio permite que este análisis pueda ser implementado en cualquier otra organización, para así poder conseguir una rápida gestión de calidad, medio ambiental, seguridad y salud ocupacional de una manera eficaz y con el cumplimento ajustado a los estándares internacionales de gestión aplicada. (Ramos, W, Ospino, My Ortiz, L., 2016).

Por lo tanto, es factible que la implementación de un Sistema Integrado de Gestión como herramienta de gestión permita conseguir clientes satisfechos, cumplimiento de la legislación laboral, uso de recursos humanos, creando trabajadores calificados y motivados.

Las empresas visitadas tomaron la decisión de certificar hace más 10 años. Las razones de atrasos o demoras en su certificación se deben a factores controlables de la empresa, y prioritariamente se deben a cambios de gerencia, falta de capital o porque no tienen mayor necesidad.

Para el mantenimiento del sistema los entrevistados respondieron que lo hacen a través de registros, acciones correctivas y preventivas, auditorías internas, reuniones semanales. 
La presente investigación se fundamenta en la conveniencia de la implementación de los Sistemas Integrados de Gestión en las organizaciones, que la puede alcanzar todo tipo de organización, también las medianas, pequeñas y microempresas, empresas de transformación o servicios, que aplicado adecuadamente es considerado como una herramienta de gestión.

\section{MÉTODOS}

Para la obtención de resultados se diseñó un estudio descriptivo, aplicado y de corte transversal, el presente estudio alcanzó a investigar a una muestra seleccionada a conveniencia, de 08 empresas de producción y servicios que están situadas en el parque industrial de Arequipa, se realizó una encuesta y una entrevista a los responsables del Sistema Integrado de Gestión, la técnica para la elaboración del cuestionario fue depurada con criterio lógico y ordenado para obtener datos primarios o de primer orden. También se utilizaron técnicas para obtener datos secundarios. Dicha investigación se realizó desde junio a noviembre del 2019. El proceso de la investigación realizada consistió en las siguientes etapas:

1. Establecer el objetivo del estudio. El objetivo específico de la encuesta era probar la hipótesis sobre el papel estratégico del contexto de una organización en el sistema integrado de gestión. El objetivo del estudio es analizar la implementación del sistema integrado de gestión.

2. Elaboración del cuestionario y aplicación. El cuestionario incluyó 17 preguntas relacionadas con el contexto de los encuestados respecto a varios aspectos de la organización con relación en los sistemas integrados de gestión.

3. Elegir el grupo objetivo de empresas y aplicarles la encuesta. Para ello se pidió el concurso de los responsables de Sistemas Integrados de Gestión. Los encuestados participaron en la encuesta y entrevista, y se recibió un cuestionario de cada uno de ellos.

4. Análisis de resultados de investigación.

5. Comentarios de los encuestados.

Para profundizar la investigación se escogió una empresa que tiene las tres certificaciones internacionales vigentes y finalmente se elaboró el informe final donde se muestran los resultados. 


\section{RESULTADOS}

Con información del Centro de Desarrollo Industrial - CDI, que es un organismo que forma parte de la Sociedad Nacional de Industrias del Perú y con el análisis de la investigación realizada, se contrastó y determinó que, de las 40 empresas asociadas al Parque Industrial de Arequipa, solo 19 empresas han implementado alguna de las tres normas internacionales, el detalle en específico es el siguiente:

$\checkmark \quad 17$ empresas tienen la certificación ISO 9001 referida a Gestión de la Calidad (Tabla 01).

$\checkmark \quad 07$ empresas cuentan con la certificación ISO 14001 referida a Gestión Ambiental (Tabla 02).

$\checkmark \quad 04$ empresas cuentan con la certificación OSHAS 18001 referida a Gestión de Seguridad y Salud Ocupacional (Tabla 03).

También se puede apreciar que 04 empresas del parque industrial de Arequipa cuentan con las tres (03) normas internacionales, 01 empresa tiene dos (02) de las tres normas internacionales y 13 empresas tienen al menos una (01) de las tres normas internacionales), asimismo doce (12) empresas cuentan únicamente con ISO 9001, dos (02) empresas cuentan únicamente con ISO 14001 y ninguna de las empresas cuenta únicamente con OSHAS 18001 (Tabla 04).

Las siguientes tres combinaciones muestran una dependencia constante en el desempeño de la empresa: ISO 9001, ISO 9001 + ISO 14001 e ISO 9001 + ISO 14001 + OHSAS 18001. Siendo ISO 9001 el factor común de estas combinaciones y también el primer estándar adoptado por la mayoría de las empresas, los resultados sugieren que podría ser un motor relevante para mejorar el desempeño de la empresa.

A continuación, se muestran las tablas 1, 2, 3 y 4 con las empresas que tienen dichas certificaciones internacionales en el parque industrial de Arequipa.

También, se muestra la tabla 5 con los resultados de las encuestas y entrevistas a los responsables del Sistema Integrado de Gestión de las empresas muestreadas del parque industrial de Arequipa. 
Tabla 1

EMPRESAS CERTIFICADAS ISO 9001

\begin{tabular}{lcc}
\hline Razón social / empresa & Fecha de última certificación & Certificadora \\
\hline ALICORP S.A.A. & 01 -may-07 & GLC \\
CORPORACIÓN ACEROS AREQUIPA S.A. - PLANTA DE AREQUIPA & feb-98 & ABS \\
CORPORACIÓN PERUANA DE PRODUCTOS QUÍMICOS & 18 -feb-2003 & SGS \\
CRUBHER / GEODRILL & 21 -mar-13 & BVQI \\
FRANKY \& RICKY S.A. & 15 -ago-2010 & SGS \\
GLORIA S.A. & 01 -jun-07 & GLC \\
LAIVE S.A. & 28 -feb-14 & BVQI \\
METALÚRGICA QUÍMICA S.R.L. & 08 -feb-07 & SGS \\
MOLY-COP ADESUR S.A.C. & $27-$-nov-98 & BVQI \\
PAPELERA PANAMERICANA S.A. & - & BUREAU \\
PRAXAIR PERÚ S.A. & 12 -feb-2013 & SGS \\
RANSA CIAALMACENERA S.A. / CASA & 25 -mar-2009 & SGS \\
SERVICIO NACIONAL DE ADIESTRAMIENTO EN TRABAJO INDUSTRIAL & $14-$-abr-03 & BVQI \\
SENATI & & \\
SGS DEL PERÚ SAC & $06-$-nov-06 & ABS \\
SOCIEDAD ELÉCTRICA DEL SUR OESTE S.A. - SEAL & 28 -feb-2012 & SGS \\
TRANSALTISA S.A. & 15 -mar-13 & BVQI \\
UNIVERSIDAD CATOLICA SAN PABLO & - & BVQI
\end{tabular}

FUENTE: Elaboración propia (2019)

Tabla 2

EMPRESAS CERTIFICADAS ISO 14001

\begin{tabular}{lcc}
\hline Razón social / empresa & Fecha de última certificación & Certificadora \\
\hline ALICORP S.A.A. & 01 -may-10 & - \\
COMPAÑÍA DE MINAS BUENAVENTURA S.A.A. & - & - \\
- UP ORCOPAMPA & 17 -ene-09 & BVQI \\
MOLY-COP ADESUR S.A. & - & - \\
PRAXAIR PERÚ S.R.L. & - & - \\
SERVICIO NACIONAL DE ADIESTRAMIENTO & 18 -nov-08 & BVQI \\
EN TRABAJO INDUSTRIAL - SENATI & 08 -may-08 & SGS \\
TRANSALTISA S.A. & & \\
YURA S.A. &
\end{tabular}

FUENTE: Elaboración propia (2019) 
Tabla 3

EMPRESAS CERTIFICADAS CON OSHAS 18001

\begin{tabular}{lcc}
\hline Razón social / empresa & Fecha de última certificación & Certificadora \\
\hline ALICORP S.A.A. - CONSUMO MASIVO & $07-$ may-10 & GLC \\
MOLY-COP ADESUR S.A. & - & - \\
SERVICIO NACIONAL DE ADIESTRAMIENTO & - & - \\
EN TRABAJO INDUSTRIAL - SENATI & $17-$-sep-09 & BVQI \\
TRANSALTISA S.A. & & \\
\hline
\end{tabular}

FUENTE: Elaboración propia (2019)

Tabla 4

RESUMEN DE EMPRESAS CERTIFICADAS

\begin{tabular}{|c|c|c|c|c|}
\hline Razón social / empresa & $\begin{array}{l}\text { ISO } \\
9001\end{array}$ & $\begin{array}{c}\text { ISO } \\
14001 \\
\end{array}$ & $\begin{array}{c}\text { OSHAS } \\
18001 \\
\end{array}$ & Total \\
\hline ALICORP S.A.A. & $x$ & $x$ & $x$ & 3 \\
\hline COMPAÑÍA DE MINAS BUENAVENTURA S.A.A. - UP ORCOPAMPA & & $x$ & & 1 \\
\hline CORPORACIÓN ACEROS AREQUIPA S.A. - PLANTA DE AREQUIPA & $x$ & & & 1 \\
\hline CORPORACIÓN PERUANA DE PRODUCTOS QUIMICOS & $x$ & & & 1 \\
\hline CRUBHER / GEODRILL & $x$ & & & 1 \\
\hline FRANKY \& RICKY S.A. & $x$ & & & 1 \\
\hline GLORIA S.A. & $x$ & & & 1 \\
\hline LAIVE S.A. & $x$ & & & 1 \\
\hline METALÚRGICA QUÍMICA S.R.L. & $x$ & & & 1 \\
\hline MOLY-COPADESUR S.A.C. & $x$ & $x$ & $\mathrm{x}$ & 3 \\
\hline PAPELERA PANAMERICANA S.A. & $x$ & & & 1 \\
\hline PRAXAIR PERÚ S.A. & $x$ & $x$ & & 2 \\
\hline RANSA CIA ALMACENERA S.A. / CASA & $x$ & & & 1 \\
\hline SERVICIO NACIONAL DE ADIESTRAMIENTO EN TRABAJO INDUSTRIAL - SENATI & $x$ & $x$ & $x$ & 3 \\
\hline SGS DEL PERÚ SAC & $x$ & & & 1 \\
\hline SOCIEDAD ELÉCTRICA DEL SUR OESTE S.A. - SEAL & $x$ & & & 1 \\
\hline TRANSALTISA S.A. & $x$ & $\mathrm{x}$ & $\mathrm{x}$ & 3 \\
\hline UNIVERSIDAD CATÓLICA SAN PABLO & $x$ & & & 1 \\
\hline YURA S.A. & & $\mathrm{x}$ & & 1 \\
\hline TOTAL & 17 & 7 & 4 & \\
\hline
\end{tabular}

FUENTE: Elaboración propia (2019) 
Tabla 5

RESULTADOS ENCUESTA Y ENTREVISTA

Tipo de actividad implementación sistema de gestión

$\begin{array}{ll}\text { Procesos } & 100 \%\end{array}$

Duración tiempo en certificarse

$\begin{array}{ll}01 \text { año } & 25 \% \\ 02 \text { años } & 50 \% \\ 03 \text { años - mas } & 25 \%\end{array}$

Motivo de certificación

Globalización $25 \%$

Exigencia clientes $\quad 37.5 \%$

Mejora procesos $\quad 25 \%$

Aumentar producción $\quad 12.5 \%$

Razones por aplazamiento de certificación

Cambio de gerencia

$24 \%$

Falta de capital

$38 \%$

No tienen necesidad

$38 \%$

Requisito inicial para certificación

$\begin{array}{ll}\text { Sensibilización y capacitación } & 100 \%\end{array}$

Mantenimiento del sistema

$\begin{array}{ll}\text { Uso registros, auditorías } & 100 \%\end{array}$

Contaron con presupuesto

$\begin{array}{ll}\text { Apoyo de gerencia } & 100 \%\end{array}$

Medición del impacto

Uso KPI

$100 \%$

FUENTE: Elaboración propia (2019) 


\section{DISCUSIÓN}

De las 8 empresas visitadas, 7 son del rubro industrial y 1 de educación, esta última nos servirá para mostrar algunos resultados.

La tabla 5 muestra los resultados obtenidos de la encuesta y entrevista a los responsables del Sistema Integral de Gestión.

- Para qué tipo de actividades han implementado un sistema de gestión, el $100 \%$ respondió que es para todos sus procesos.

Los resultados del estudio demuestran una línea de acción en obtener un ISO. Todos los requisitos de esta Norma Internacional son genéricos y se pretende que sean aplicables a todas las organizaciones, sin importar su tipo o tamaño, o los productos y servicios suministrados. (ISO 9001:2015(es), 2019).

- A las empresas encuestadas les tomó el tiempo de un 1año (25\%); 2 años (50\%); de 3 años a más (25\%) la certificación.

Tras revisar la evidencia científica disponible hasta la fecha, no se ha encontrado datos sobre la equivalencia. "El tiempo para la certificación del sistema es variable en función del tamaño de la organización y de la complejidad de sus procesos o prestación del servicio. El tiempo mínimo estimado en condiciones normales, para una PYME es 4 a 6 meses pudiendo ser menor en función de la dedicación a los recursos de la organización". (Gómez, I., 2019).

- El motivo que llevaron a certificarse fue: la globalización (25\%), exigencias de clientes (38\%); mejora de procesos (24\%); aumentar volumen de producción (13\%).

Este estudio no encontró ninguna relación en la literatura del motivo que los llevaron a certificarse. "El objetivo es encontrar información para mejorar la calidad de los productos y servicios $(77.5 \%)$ y formar la base para el desarrollo de políticas y objetivos en el campo de la calidad (45.0\%). También, los encuestados notaron una evaluación del nivel de competitividad de la organización (32.5\%) y un Análisis del sistema de gestión de la calidad por parte de la dirección (22,5\%). (Makolov, V., 2019). 
- El total de las empresas visitadas tomaron la decisión de certificar hace más de 10 años. Las razones que han atrasado este proceso se deben a factores controlables de la empresa como el cambio de gerencia (24\%), la falta de capital (38\%); no tienen mayor necesidad (38\%).

Esta investigación halló que el peso económico prima sobre la mejora de los sistemas de calidad. Hay una relación entre el cambio de gerencia y capital. "Las normas ISO aportan grandes beneficios en el sistema de calidad a las empresas, pero, aunque ella está diseñada para agregar valor en el sistema de calidad, no siempre se cumple el objetivo, no por causa de la misma norma”. (Martínez, J., 2019).

- Todos los responsables del Sistema de Gestión indicaron que tuvieron que empezar con un plan de sensibilización y un programa permanente de capacitación.

La literatura existente ha encontrado una fuerte relación entre este resultado con otros estudios. "En la versión 1994 de la norma ISO 9000, las directrices y orientaciones que complementan la norma, contenidas en el punto 5.2.4 de ISO 9004, indicaban que sería conveniente determinar el nivel de competencia, experiencia y capacitación necesarios para asegurar la capacidad laboral del personal". (Gestiopolis, 2019).

- Para el mantenimiento del sistema los entrevistados respondieron que lo hacen a través de registros, acciones correctivas y preventivas, auditorías internas, reuniones semanales.

Haciendo alusión al mantenimiento, esta investigación encontró relación con otros estudios. "Desarrollar e implementar métodos de mantenimiento, para asegurarse de que la infraestructura continúe cumpliendo las necesidades de la organización; estos métodos deberían considerar el tipo y frecuencia del mantenimiento y la verificación de la operación de cada elemento de la infraestructura, basado en su criticidad y en su aplicación” (Mantenimiento Fácil, 2019).

- Todas las empresas encuestadas contaron con el presupuesto suficiente para su implementación.

Los resultados de esta investigación muestran un alto compromiso presupuestal. "El costo de la implementación depende de tres factores: 
1) El primero, por supuesto, es cuán grande es su compañía, esto tendrá una gran influencia en el costo del proyecto.

2) El segundo es también bastante importante y es qué tipo de sistema de calidad tiene en uso actualmente. Si actualmente cuenta con un buen sistema, su trabajo para estar en conformidad con ISO 9001 será menor.

3) Otros miembros de su compañía durante la semana para dedicarle al proyecto y la necesidad de un asesor.". (Normas9000, 2019).

- Con relación a la medición del impacto respondieron que utilizan indicadores de rentabilidad, indicadores de medición de satisfacción del cliente; evaluación de proveedores.

Estudios previos han señalado la importancia del uso de indicadores para medir los objetivos y los resultados. "Entre los beneficios principales de los sistemas integrados de gestión, están la racionalización de la documentación, la armonización de los criterios referidos a la organización, medición y seguimiento de los procesos; así como el logro de una mayor eficiencia en la toma de decisiones por la dirección, al disponer de una visión global de los sistemas. Todo ello contribuye a la mejora de la capacidad de reacción de la organización, frente a las nuevas necesidades o expectativas de las partes interesadas". (Antúnez Saiz, 2016)

- Las conclusiones de este estudio muestran que de las 8 empresas contempladas en este estudio el $87.5 \%$ son empresas industriales y el $12.5 \%$, de educación. En el estudio realizado se ha constatado que solo existen 19 empresas del parque industrial de Arequipa -representa el 0.014\% del total de empresas del departamento- han implementado alguna de las tres normas optativas internacionales. Se ha evidenciado que las empresas certificadas obtienen mayor valor que las que no lo están. En la evaluación realizada las empresas señalan que el motivo que las llevaron a certificarse fue la globalización, exigencias de clientes, mejora de procesos, aumentar volumen de producción entre los factores más importantes. El total de las empresas visitadas, afirmaron que la decisión de certificarse la iniciaron hace más de 10 años. Y las razones de peso que argumentan es que el atraso o demora se deben a factores controlables de la empresa, que van desde el cambio de gerencia, la falta de capital, y que no tienen mayor necesidad. 
- Todos los responsables del Sistema de Gestión, indicaron que tuvieron que empezar con un plan de sensibilización y un programa permanente de capacitación. Es necesario hacer un proceso de sensibilización que involucre a todos los actores de la empresa; para llevar a cabo la implementación del mismo, la sensibilización debe ser más que la implementación; debe ser un proceso de facilitación y de concientización hacia el cambio; el cual aportará elementos que creen un ambiente favorable para el nuevo sistema de calidad en la empresa.

- Los resultados confirman que las empresas con alguna certificación tienen mejores condiciones y mejores procesos de gestión que las empresas no certificadas. En la muestra resalta la importancia y los efectos positivos de la certificación en el nivel de calidad de las empresas del parque industrial de Arequipa. Los principales beneficios percibidos en la implementación del sistema de gestión para las empresas situadas en el parque industrial de Arequipa incluyen documentación mejorada, eficiencia mejorada del sistema de calidad y una selección de proveedores más efectiva. Asimismo, la conciencia ambiental se ha vuelto cada vez más pronunciada entre las diversas partes interesadas, como los gobiernos y las comunidades, muchas empresas comienzan a adoptar un enfoque más respetuoso con el medio ambiente en sus operaciones. Incluso una industria orientada a servicios como la educación no es una excepción. Acerca del mantenimiento del sistema integrado de gestión, los entrevistados respondieron que lo hacen a través de registros, acciones correctivas y preventivas, auditorías internas, reuniones semanales. Se recomienda la realización de nuevos estudios, sería interesante comparar el impacto de la certificación ISO en la mano de obra. productividad en otras áreas geográficas. Además del análisis a nivel de país o un nivel de industria.

\section{Caso real: SENATI Sistema Integrado de Gestión -Gestión de la Calidad, Ambien- tal y Seguridad y Salud Ocupacional. (Senati, 2019)}

Objetivos Principales del Sistema Integrado de Gestión

- Satisfacer necesidades y expectativas de los Clientes.

- Prevenir la contaminación ambiental, usar racionalmente los recursos y manejar responsablemente los residuos.

- Prevenir los riesgos de seguridad y salud en el trabajo. 


\section{¿Por qué adoptar un Sistema Integrado de Gestión?}

- Se le considera una buena herramienta de gestión.

- Asegura el control de los factores que determinan el cumplimiento de objetivos en función de la Política, Misión y Visión Institucionales.

- Siendo voluntaria la adopción de un Sistema Integrado de Gestión, al hacerlo nos obligamos al cumplimiento legal correspondiente.

- En el caso del sistema de seguridad y salud ocupacional, la adopción es obligatoria por disposición legal.

\section{Procesos Principales}

Dirección. - política; planeamiento estratégico; planeamiento operativo y presupuesto; marketing; revisión

Operativos. - diseño y desarrollo técnico pedagógico; prestación del servicio y post venta (bolsa de trabajo)

Soporte. - (Tecnología de la información; gestión de recursos humanos; administración de bienes; abastecimiento; procesos financieros; proceso de relación con el cliente; auditorías internas; comunicación interna y externa; prevención de riesgos y caso de emergencia.

Modelo del Sistema de Gestión Basado en Procesos

- Empieza en clientes y termina en clientes, mejora continua en los procesos de gestión.

\section{El Mayor Impacto del SIG-SENATI}

Siendo el SENATI, una institución de Formación y Capacitación Profesional, el mayor y más importante impacto se da en las empresas en las que se insertan nuestros egresados, ya que en ellas los aspectos de calidad, cuidado ambiental y seguridad y salud de las personas son más necesarios tenerlos bajo control, de manera que se propicie su mejoramiento continuo.

"El Sistema de Gestión Integrado del SENATI, que aplica las normas internacionales ISO 9001 de Gestión de la Calidad, ISO 14001 de Gestión Ambiental y OHSAS 18001 de Seguridad y Salud Ocupacional, tiene como uno de sus principios rectores la satisfacción de las necesidades y requisitos de los clientes de la organización" (oitcintenfor.org, 2019). 


\section{REFERENCIAS}

Albulescu, C. T., Drăghici, A., Fistiş, G. M., \& Truşculescu, A. (2016). Does ISO 9001 Quality Certification Influence Labor Productivity in EU-27? Procedia - Social and Behavioral Sciences, 221, 278-286. https://doi.org/10.1016/j.sbspro.2016.05.116

Antúnez Saiz, V. I. (2016). Sistemas integrados de gestión:: de la teoría a la práctica empresarial en Cuba. Cofin Habana, 10(2), 1-28.

Asociación de Empresas del Parque Industrial de Arequipa - ADEPIA (10 de diciembre del 2019). Directorio de empresas. Recuperado de: http://www.adepia.com.pe/listings-column-map/

Calidad ISO9001, (11 de diciembre del 2019). "Definición de términos". Recuperado de: http://iso9001calidad.com/definicion-determinos-586.html

Centro de Desarrollo Industrial - CDI, (18 de diciembre del 2019). Empresas certificadas. Recuperado de: http://www.cdi.org.pe/asist_empcertISO9000-020.htm

Evangelos Psomas \& Jiju Antony (2015) The effectiveness of the ISO 9001 quality management system and its influential critical factors in Greek manufacturing companies, International Journal of Production Research, 53:7, 2089-2099, DOI: 10.1080/00207543.2014.965353

Gestiopolis, (22 de noviembre del 2019). Capacitación efectiva según la Norma ISO 90012000. Recuperado de: https:/www.gestiopolis.com/capacitacion-efectiva-segun-la-norma-iso9001-2000\%.

Geromin, A. N., \& Galleguillo, D. N. (2015, January 1). Integrated Management Systems (OHSAS 18001 , ISO 14001 \& 9001) in SMEs. American Society of Safety Engineers.

Gómez, Ignacio, (20 de diciembre del 2019). Preguntas Frecuentes ISO 9001:2008. Recuperado de: http://www.hederaconsultores.com/docs/Preguntas_frecuentes_ISO_9001.pdf

Instituto Nacional de Estadística e Informática INEI. (2019). Perú: Estructura Empresarial 2018 Recuperado de [Consulta: 06 de enero del 2020]. https://www.inei.gob.pe/media/ MenuRecursivo/publicaciones digitales/Est/Lib1703/

ISO 9001:2015(es), (28 de diciembre del 2019). Sistemas de gestión de la calidad — Requisitos. Recuperado de:https://www.iso.org/obp/ui/es/\#iso:std:iso:9001:ed-5:v1:es

ISO Survey. (2019). THE ISO SURVEY OF MANAGEMENT SYSTEM CERTIFICATIONS 2018 - EXPLANATORY NOTE Background The ISO Survey of Certifications is an annual survey of the number of valid certificates to (Issue September).

ISO TOOLS, (10 de diciembre del 2019). “¿Qué es la integración de sistemas de gestión?” Recuperado de: https://www.isotools.org/normas/sistemas-integrados/

Jiménez, Daniel. (11 de diciembre del 2019). "¿Cómo implementar un sistema de gestión de calidad?". Recuperado de: https://www.pymesycalidad20.com/\%e2\%96\%b7comoimplementar-un-sistema-de-gestion-de-calidad.html

Makolov, Vasily. (2019). Context of Organization and Quality Management. IOP Conference Series: Earth and Environmental Science. 272.032216. 10.1088/1755-1315/272/3/032216. 
Walter F. Deza-Loyaga, Jorge A. Aparicio-Ballena, Jenry A. Hidalgo-Lama

Mantenimiento Fácil, (28 de noviembre del 2019). ISO 9000. Recuperado de: http://www.manteni mientofacil.com.ar/iso.htm.

Martínez, Jonny (14 de noviembre del 2019). Normas ISO. Recuperado de: https://www.liderazgo ymercadeo.co/normas-iso/

Normas9000, (29 de noviembre del 2019). Preguntas Frecuentes. Recuperado de: http://www.normas9000.com/content/FAQ.aspx\#pregunta13.

Oitcinterfor, (15 de diciembre del 2019). Evaluación del impacto de la formación profesional industrial realizada por el SENATI en el Perú. Recuperado de:http://guia.oitcinterfor.org/sites/ default/files/experiencias/Peru_Evaluacion_impacto_FP__SENATI.pdf.

Ramos, W., Ospino, M., \& Ortiz, L. (2016). "Análisis del sistema integrado de gestión en una empresa calzado”, Investigación y Desarrollo en TIC, vol. 7, no. 2, pp. 74-85.

SENATI, (05 de diciembre del 2019). SG_presentaciones. Recuperado de: http://intranet.senati. edu.pe/SG_presentaciones.asp 


\section{ANEX01.}

Encuesta utilizada para llevar a cabo las entrevistas en empresas certificadas de algún Sistema de Gestión

IMPORTANTE. - La presente encuesta responde al estudio del impacto de la certificación ISO 9001, ISO 14001 y OSHAS 18001 en empresas arequipeñas que han logrado dicha certificación.

\section{DATOS GENERALES}

1. Razón social:

2. Dirección:

3. Teléfono:

4. Actividad principal:

5. Responsable del Sistema de Gestión:

II. PERSONAQUELLENALAENCUESTA

1. NombreyApellidos:

2. Cargo:

3. E-mail:

\section{CUESTIONARIODEPREGUNTAS}

1. ¿Para qué actividades han implementado un sistema de gestión?

2. ¿Hace cuánto tiempo tomaron la decisión de certificarse?

3. ¿Cuánto es tiempo estimado por la empresa para certificarse?

4. ¿Cómo conoció los sistemas de gestión?

5. ¿Ha efectuado cursos de formación relacionados a los sistemas de gestión?

6. ¿Cuál fue el motivo de la certificación?

7. ¿En qué año consiguieron la certificación?

8. ¿Cuánto tiempo duro el proceso de certificación?

9. ¿Tiene resultados de la implantación y mantenimiento del sistema de gestión?

10. ¿Desde la obtención de la certificación qué beneficios han obtenido en cuanto a nuevos mercados, nuevos clientes?

11. ¿La implantación del sistema de gestión en la organización ha generado cambios trascendentales?

12. Con la implantación del sistema de gestión, ¿qué se ha conseguido? 
13. ¿Contó la organización con el presupuesto suficiente para su implementación?

14. ¿Cuál cree usted, que son las razones porque las empresas no implementan sistema de gestión?

15. En el mantenimiento del sistema, ¿qué dificultades se presentaron?

16. ¿Cómo se hace seguimiento luego de la implementación del sistema?

17. ¿Su empresa está dispuesta a difundir su experiencia, para que otras organizaciones logren la certificación de un sistema de gestión?

NOTA:

Los resultados se darán a conocer una vez concluido el estudio, el mismo que permitirá elaborar una guía para que otras organizaciones puedan iniciar el proceso de certificación. 